\title{
Understanding How Chorea Affects Health-Related Quality of Life in Huntington Disease: An Online Survey of Patients and Caregivers in the United States
}

\author{
Eileen Mack Thorley ${ }^{1} \cdot$ Ravi G. Iyer ${ }^{2} \cdot$ Paul Wicks $^{1} \cdot$ Chris Curran $^{1} \cdot$ Sanjay K. Gandhi $^{2} \cdot$ \\ Victor Abler $^{2} \cdot$ Karen E. Anderson ${ }^{3} \cdot$ Noelle E. Carlozzi ${ }^{4}$
}

Published online: 11 May 2018

(c) The Author(s) 2018

\begin{abstract}
Background Chorea is the hallmark motor feature of Huntington disease (HD) and can negatively impact daily functioning and health-related quality of life (HRQoL).

Objective The objective of this study was to evaluate how chorea impacts HRQoL and overall functioning among HD patients participating on the PatientsLikeMe website (www.PatientsLikeMe.com).

Methods A survey was provided to HD participants and/or their caregivers via PatientsLikeMe (9 February 2017-22 March 2017), comprising multiple-choice and open-ended questions designed to assess how chorea impacts HRQoL and overall functioning, and the importance of treating chorea. The HDQLIFE measurement system was used to evaluate patient-reported outcomes of chorea and compare Anxiety and Stigma scores in participants with high chorea versus those with low chorea [HDQLIFE Chorea scores $\geq 60(n=45)$ vs. $<60(n=38)]$.
\end{abstract}

Electronic supplementary material The online version of this article (https://doi.org/10.1007/s40271-018-0312-x) contains supplementary material, which is available to authorized users.

Eileen Mack Thorley

emackthorley@patientslikeme.com

1 PatientsLikeMe, 160 2nd Street, Cambridge, MA 02142, USA

2 Teva Pharmaceutical Industries, 41 Moores Rd, Frazer, PA 19355, USA

3 Department of Psychiatry, Georgetown University, Washington, DC, USA

4 Department of Physical Medicine and Rehabilitation, University of Michigan, 500 S. State Street, Ann Arbor, MI 48109, USA
Results A total of 115 participants ( $n=35$ caregivers; $n=80$ individuals with HD) were included in this study. Among those experiencing chorea $(n=83,74 \%$ of respondents), $66 \%$ indicated it was 'Very Important' to manage chorea; however, only $47 \%$ agreed that their current medication regimen helped manage their movements. In general, respondents reported that chorea negatively affected HRQoL [HDQLIFE Chorea mean score (standard deviation): 59.3 (6.1)]. Consistent with this, significantly higher Anxiety $(P=0.0423)$ and stigma $(P<0.0001)$ scores were observed among respondents with high chorea than in those with low chorea.

Conclusions These results highlight the negative impact of chorea on HRQoL and overall functioning in individuals with HD. Better chorea treatment options are needed to successfully manage symptoms and to help improve HRQoL in these individuals, and patient experiences of anxiety and stigma should be considered in treatment plans.

\section{Key Points for Decision Makers}

Our study highlights the negative impact of chorea [the most prominent motor feature of Huntington disease (HD)] on quality of life in patients with HD.

Better treatment options are needed to successfully manage symptoms of chorea and to improve quality of life in these individuals. 


\section{Introduction}

Huntington disease (HD) is a hereditary, multifaceted neurodegenerative disorder characterized by motor dysfunction, progressive cognitive impairment, and emotional-behavioral symptoms [1-3]. HD has a profound impact on daily life across physical/functional, cognitive, emotional, and social domains for affected patients, with increasing challenges as the disease progresses $[4,5]$. There is no cure for the disease, but one of the defining motor symptoms in patients living with HD, choreawhich is characterized as abnormal, abrupt, irregular, unpredictable, and non-stereotyped movements - can be treated pharmacologically [6, 7] and supported with physical therapy [8].

As the hallmark neurological feature of $\mathrm{HD}$, chorea is present in $90 \%$ of patients with adult-onset HD [7, 9]. It has been shown that chorea may negatively impact health-related quality of life (HRQoL) by increasing the risk of injury, interfering with daily functioning, and causing difficulty in performing motor tasks at the workplace [7].

As of the date of publication, only two medications have been approved for the treatment of chorea associated with HD. Tetrabenazine [Xenazine ${ }^{\circledR}$, Ovation Pharmaceuticals (Lundbeck, Deerfield, Ill), approved by the US Food and Drug administration (FDA) in 2008] was the only treatment option approved by the FDA for HD chorea at the time of this study. Tetrabenazine is associated with adverse effects such as depression and suicidal ideation (resulting in a Black Box warning), sedation/somnolence, insomnia, akathisia, and parkinsonism [10,11]. While tetrabenazine was efficacious in improving chorea in patients with HD, whether it can improve HRQoL is unknown. More recently, deutetrabenazine, a novel vesicular monoamine transporter 2 inhibitor, was approved for the treatment of chorea associated with HD (Austedo ${ }^{\circledR}$; Teva Pharmaceutical Industries, North Wales, PA, USA, approved on 3 April 2017) [12, 13]. Other treatment options have poorly understood benefits and are used off-label despite limited evidence supporting their use [14].

While clinically observed measures such as the Unified Huntington's Disease Rating Scale (UHDRS) remain the gold standard outcome for clinical trials in HD chorea, the real-life experience of patients living with HD and their caregivers may provide additional relevant information on the impact of this disease. It has been suggested that the stigma associated with chronic neurological disorders is high and impacts HRQoL, especially in patients with visible motor symptoms, such as those with epilepsy, multiple sclerosis (MS), and Parkinson disease [7, 15-17]. In HD, chorea is likely to contribute to embarrassment and social isolation of affected individuals and their families [5, 7]. In addition to patients with $\mathrm{HD}$, their caregivers also carry a heavy burden. For example, caregivers may need to subordinate their own needs to those of the patient and their caregiving role, and thus their HRQoL is greatly and negatively impacted $[18,19]$.

In the current study, we aimed to explore how chorea impacts HRQoL and overall functioning among a population of individuals with HD. We recruited participants from a patient-powered, data-driven network, PatientsLikeMe (www.patientslikeme.com), a free online community of more than 500,000 members who have over 2700 conditions. The site enables members to monitor symptoms of their condition(s), share their disease experiences and treatment outcomes, and learn how to improve their care through peer-to-peer interactions [20]. The potential of PatientsLikeMe to provide help to patients with other neurological conditions, such as amyotrophic lateral sclerosis, Parkinson disease, MS, and epilepsy, has been described previously [20, 21]. Using survey and questionnaire measures, we aimed to evaluate the experience of individuals with $\mathrm{HD}$ and understand other important factors affecting chorea treatment, including total functional capacity and quality of life, based on responses of individuals with $\mathrm{HD}$ and/or caregivers who answered the questions from the perspective of those for whom they cared.

\section{Methods}

\subsection{Participant Recruitment and Survey Development}

To obtain real-world perspectives of patients living with HD, a cross-sectional survey was developed and administered to PatientsLikeMe members who reported HD as their condition. To guide survey development, six 45- to 60-min semi-structured interviews were conducted with PatientsLikeMe members with $\mathrm{HD}$, by phone or video conference, to better understand their experience with chorea and identify factors important to patients and caregivers regarding treating chorea. Two of these six interviews included patient-caregiver dyads, also reflecting both patients' and caregivers' perspectives on HD. Based on a qualitative review of the interviews using content analysis [22, 23], themes included emotional burden, anxiety, stress, and depression. Interviewees described the impact of HD on social life, feelings of embarrassment, and approaches to coping and 'health hacks'. These themes, along with a review of the literature and input from key opinion leaders (authors Dr. Noelle Carlozzi and Dr. Karen Anderson), were used to guide selection of measures included in the study. 
The survey was fielded to members of PatientsLikeMe on its Open Research Exchange platform if they were over 18 years of age, resided in the USA, and reported HD as one of their health conditions or indicated being a caregiver of a patient with HD. The invitation to participate in the survey contained a brief explanation of the survey and a link to participate. Users who did not complete the survey within 3 days were sent an e-mail reminder to encourage completion. In addition, external recruitment was also conducted using digital advertising optimized to maximize sign-up of new members with the desired condition. After users registered for PatientsLikeMe, they were invited to participate in the research study according to the same method described. The survey was fielded via PatientsLikeMe to participants with HD and/or their caregivers from 9 February 2017 to 22 March 2017. Licensing agreements were obtained for each validated instrument included in the survey.

\subsection{Survey and Questionnaire Measures}

This study utilized a combination of custom survey questions and validated questionnaires. The survey comprised multiple-choice and open-ended questions designed to assess how chorea impacted HRQoL and overall functioning, patient experience with treating chorea, and aspects of managing chorea important to patients and caregivers (see Electronic Supplementary Material Tables 1 and 2 for representative survey questions about managing chorea and how chorea affects patients). Caregivers completed survey questions from the perspectives of those for whom they cared. Survey respondents were asked if they, or the individuals they were caring for, were currently experiencing chorea. A definition of chorea was provided that used words patients referenced during the semi-structured interviews (e.g. 'floppy', 'involuntary swaying') to supplement both the clinical description of chorea as an "involuntary, irregular, and often sudden or jerky movement that may involve the trunk, arms or legs, and face" and input from the PatientsLikeMe internal clinical team of healthcare professionals.

\subsubsection{Total Functional Capacity (TFC) Score}

To characterize disease stage and provide context for results, the five stages of HD and five levels of function were assessed using a self-reported version of the UHDRS total functional capacity (TFC) [24]. TFC scores range from 0 to 13, with a higher score indicating better functioning, and are categorized as follows: 11-13 (stage I, least severe); 7-10 (stage II); 3-6 (stage III); 1-2 (stage IV); and 0 (stage $\mathrm{V}$, most severe) $[25,26]$. In addition, some analyses were based on two separate groups: 'early
HD' reflected by TFC scores from 7 to 13 (stages I and II) and 'late HD' reflected by TFC scores from 0 to 6 (stages III, IV, and V) [27]. Items in the self-reported version of the TFC underwent wording adjustment from the clinicianadministered version to create more complete questions and answers on the form [24].

\subsubsection{Desire to Participate in Medical Decision Making (DPMD) Score}

The Desire to Participate in Medical Decision Making (DPMD) scale, a brief, reliable, and valid measure that can be adapted to any condition [28], was administered to assess patient engagement in their medical care. This DPMD instrument was intended to complement custom survey questions around treatment experiences with chorea. Scores were linearly transformed on a scale from 0 to 100 , with a higher score indicating a greater desire to participate in medical decision-making [28].

\subsubsection{HDQLIFE Chorea}

The HD HRQoL measurement system (HDQLIFE) [29], including HDQLIFE Chorea [30], was used to evaluate the impact of chorea on HRQoL. For the purposes of this study, we administered [29] the six-item short form of HDQLIFE Chorea, in addition to three items from the long-form full HDQLIFE Chorea item bank. Scores indicate how a patient is functioning relative to other individuals with $\mathrm{HD}$ and is measured on a $T$-score metric, which enables comparison of scores relative to others in the population, with 50 as the mean in the reference population and 10 as the standard deviation (SD) of that population $[29,30]$. A higher score indicates more self-reported chorea. HDQLIFE scores $\geq 60$ indicate an individual reporting significant concerns that may warrant clinical follow-up due to the impact of chorea on physical and/or social functioning [30]. Reliability and validity data support the use of this measure in HD [31].

As a custom survey question apart from HDQLIFE, participants were also asked to rate the importance of managing this symptom and their level of agreement that their current medications help manage their chorea.

\subsubsection{Other HDQLIFE Measures}

HDQLIFE [29] also includes generic domains from the Patient-Reported Outcomes Measurement Information System (PROMIS)/Quality of Life in Neurological Disorders (Neuro-QoL) measurement system, which was developed [32] as a standard patient-reported outcomes (PRO) measurement tool to assess patient physical, 
emotional, cognitive, and social function [33]. As a part of this study, the Anxiety (to assess patient-reported anxiety), Stigma (to assess patient perceptions of stigma), and Positive Affect and Well-Being (to assess overall emotional well-being) [32, 34] short forms were administered. Resulting scores are on a $T$-score metric, with higher scores on Anxiety and Stigma indicating worse functioning (i.e., more anxiety and more perceived stigma, respectively), whereas higher scores on Positive Affect and Well-Being indicate better functioning [35].

\subsection{Data Analysis}

Descriptive statistics were used for respondent demographics and across all survey questions. Summary statistics for continuous variables included the number of respondents, mean, $\mathrm{SD}$, and standard error of the mean. Median and interquartile range (IQR) were used for nonnormally distributed variables. Categorical variables were summarized as frequency and percentage. $95 \%$ confidence intervals of the summary statistics were presented where appropriate. Scores for each validated measure included in the survey were calculated according to established scoring guidelines and are presented. Data for open-text survey (Electronic Supplementary Material Tables 1 and 2) responses were reviewed for patterns and themes, and qualitatively coded with a content-analysis approach [23]. The natural distribution of scores was reviewed by examining histograms, mean, SD, median, and range of the data. Anxiety, Stigma, Positive Affect and Well-Being, and DPMD scores were categorized into two subgroups of individuals with HD and experiencing chorea: those with high chorea (HDQLIFE Chorea scores $\geq 60, n=45$ ) and those with low chorea (HDQLIFE Chorea scores $<60$, $n=38$ ). Stratified analyses were conducted to obtain the descriptive statistics (mean, median) in each subgroup. Group differences for Chorea scores above and below 60 were assessed using paired $t$ tests.

In addition to subgroup analyses, we analyzed whether HDQLIFE Chorea was correlated with any of the PROMIS/Neuro-QoL measures or self-reported TFC. As a further evaluation of observed associations, a multivariable linear regression model was employed to assess the effect of PROMIS/Neuro-QoL measures and self-reported TFC (independent variables) on HDQLIFE Chorea (dependent variable). Based on visual inspection of a normal probability plot, the residuals for the dependent variable, HDQLIFE Chorea, appeared to be normally distributed. Review of the Kolmogorov-Smirnov goodness-of-fit test agreed with review of the normal probability plot in that the null hypothesis that the data follow a normal distribution was not rejected $(D=0.57, P=0.150)$. Finally, given this study was a one-time cross-sectional survey and no estimates were evaluated over time, the errors were deemed to be independent. Non-constant variance was assessed by reviewing a plot of the residuals against the predicated values, which appeared to indicate homogenous distribution of the error variance with no apparent pattern in the residuals. The variance inflation factor (VIF) was reviewed in the final model to detect possible multicollinearity among the independent variables. No variables had a VIF higher than 2, an acceptable level as it was below a value of 10 [22]. The alpha level was set to 0.05 and all analyses were run in SAS $^{\circledR} 9.4$ (SAS Institute, Cary, NC, USA).

\section{Results}

\subsection{Demographic and Clinical Characteristics of Participants}

A total of 191 invitees participated in the survey out of 270 invitations viewed (participation rate $71 \%$ ). The survey was completed by 115 participants, with 80 individuals in the HD cohort and 35 in the caregiver cohort (Fig. 1). Most participants in the HD cohort were female $(75 \%)$; approximately half of those in the caregiver cohort were male $(51 \%)$. Most participants were $\geq 24$ years old $(98 \%)$, with a mean age of 47 years. Compared with the HD cohort, the caregiver cohort was older (mean age of

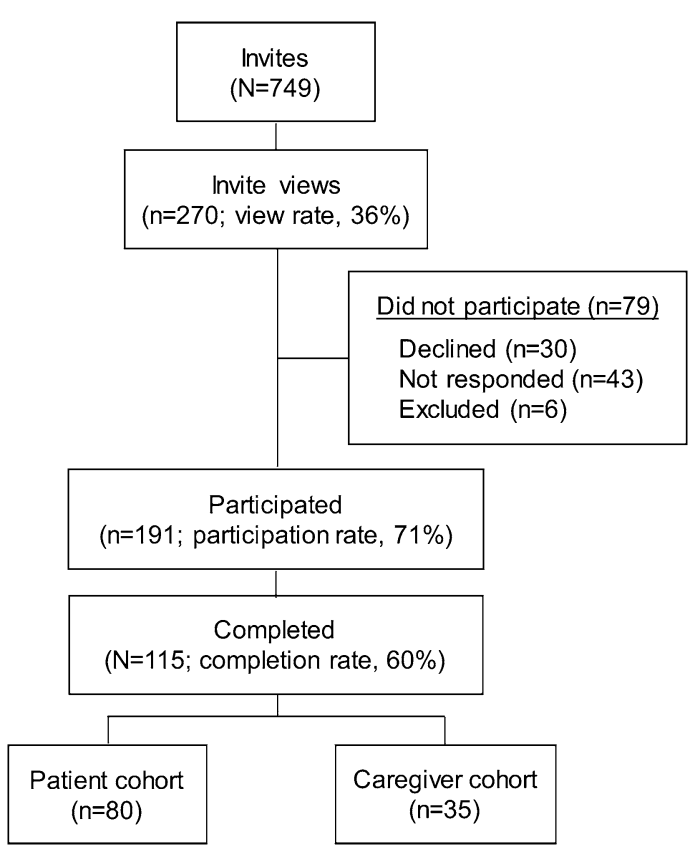

Fig. 1 Respondent selection flowchart. Among 191 individuals who participated, a total of 115 participants completed the survey, with 80 individuals with HD (HD cohort) and 35 caregivers (caregiver cohort). $H D$ Huntington disease 
51 years in the caregiver cohort vs. 45 years in the HD cohort) and reported longer duration of HD since diagnosis (median duration: 6 years in the caregiver cohort vs. 3 years in the HD cohort) (Table 1). The majority of participants in both cohorts were white $(99 \%$ in the HD cohort vs. $90 \%$ in the caregiver cohort).

\subsection{TFC Scores}

Self-reported TFC scores were reviewed to provide a measure of disease severity. Among survey respondents who completed TFC assessments $(n=112)$, the average TFC score was 6.3, indicating stage III disease. TFC scores were then stratified by HD and caregiver cohort for comparison between the two groups. Results indicated a less severe disease stage reported by individuals in the HD cohort (mean TFC score 7.5; stage II) than caregiver respondents who completed the survey on behalf of the individuals they cared for (mean TFC score 3.8, disease stage III) (Table 2). Approximately $70 \%$ of the survey population $(n=83)$ reported experiencing chorea (either themselves or the individuals they were caring for), according to the definition provided, and a lower TFC score was observed in individuals who reported experiencing chorea than in those who did not $[5.0(n=83)$ and 10.3 $(n=29)$, respectively], indicating a lower level of functioning, on average, among individuals who self-reported having chorea.

\subsection{Patient Experience with Chorea}

\subsubsection{Patient Perception of the Importance of Managing Chorea}

Patients were first asked how important it was to control or manage chorea. Among respondents who shared having experienced chorea $(72 \%, n=83)$, most indicated that it was 'very important' (59\% in the HD cohort and $71 \%$ in the caregiver cohort) to manage chorea, with $82 \%$ in the HD cohort and $94 \%$ in the caregiver cohort indicating that

Table 1 Demographic and clinical characteristics of respondents

\begin{tabular}{|c|c|c|c|c|c|}
\hline & Total $(N=115)$ & Patients $(n=80)$ & Caregivers $(n=35)$ & Test statistic ${ }^{\mathrm{a}}$ & $P$ value $^{\mathrm{b}}$ \\
\hline \multicolumn{6}{|l|}{$\operatorname{Sex}^{\mathrm{c}}[\%(n)]$} \\
\hline Female & $67 \%(76)$ & $75 \%(59)$ & $49 \%(17)$ & \multirow[t]{2}{*}{$\chi^{2}=7.44$} & \multirow[t]{2}{*}{$<0.01$} \\
\hline Male & $33 \%(38)$ & $25 \%(20)$ & $51 \%(18)$ & & \\
\hline \multicolumn{6}{|l|}{ Age (years) $[\%(n)]$} \\
\hline $19-24$ & $2 \%(2)$ & $1 \%(1)$ & $3 \%(1)$ & \multirow[t]{4}{*}{$\chi^{2}=5.29$} & \multirow[t]{4}{*}{0.15} \\
\hline $25-39$ & $35 \%(40)$ & $41 \%(33)$ & $20 \%(7)$ & & \\
\hline $40-54$ & $33 \%(38)$ & $31 \%(25)$ & $37 \%(13)$ & & \\
\hline$\geq 55$ & $30 \%(35)$ & $26 \%(21)$ & $40 \%(14)$ & & \\
\hline Years since patient diagnosis ${ }^{\mathrm{d}}$ [median (IQR)] & $4(2-9)$ & $3(2-9)$ & $6(3-13)$ & $W=2461.5$ & $<0.01$ \\
\hline \multicolumn{6}{|l|}{$\operatorname{Race}^{\mathrm{d}}[\%(n)]$} \\
\hline White & $96 \%(100)$ & $99 \%(73)$ & $90 \%(27)$ & \multirow[t]{4}{*}{$\chi^{2}=7.97$} & \multirow[t]{4}{*}{$<0.05$} \\
\hline Black & $2 \%(2)$ & 0 & $7 \%(2)$ & & \\
\hline Mixed race & $1 \%(1)$ & $1 \%(1)$ & 0 & & \\
\hline Hawaiian & $1 \%(1)$ & 0 & $3 \%(1)$ & & \\
\hline \multicolumn{6}{|l|}{ Ethnicity $^{\mathrm{c}}[\%(n)]$} \\
\hline Not Hispanic & $95 \%(94)$ & $96 \%(65)$ & $94 \%(29)$ & \multirow[t]{2}{*}{$\chi^{2}=0.18$} & \multirow[t]{2}{*}{0.67} \\
\hline Hispanic & $5 \%(5)$ & $4 \%(3)$ & $6 \%(2)$ & & \\
\hline
\end{tabular}

Percentages may not sum to $100 \%$ due to rounding

$\chi^{2}$ Chi-square, IQR interquartile range

${ }^{\mathrm{a}} \chi^{2}$ was used to analyze categorical variables. Non-normal continuous variables (years since patient diagnosis) were analyzed with Wilcoxon test under normal approximation

${ }^{\mathrm{b}}$ Two-sided $P$ value was reported

${ }^{\mathrm{c}}$ Sex, race, and ethnicity were taken from the patient profile. One respondent did not provide a sex; 11 respondents did not provide a race; 16 respondents did not provide an ethnicity. For caregivers, please note it is difficult to discern from member profiles if the ethnicity reporting reflects strictly the patient or the caregiver

${ }^{\mathrm{d}}$ Respondents provided year of diagnosis. Duration was calculated by subtracting the year of diagnosis from the year the survey was completed (2017) 
Table 2 Descriptive data for self-reported total functional capacity

\begin{tabular}{lll}
\hline TFC scale & Patient cohort $(n=77)$ & Caregiver cohort $(n=35)$ \\
\hline Total score [mean (SD)] & $7.5(3.5)$ & $3.8(3.0)$ \\
Disease stage based on TFC (\%) & & \\
Earlier stage (TFC score 7-13) & 60 & 14 \\
Later stage (TFC score 0-6) & 40 & 86 \\
\hline
\end{tabular}

Three patient respondents were missing across the TFC items and thus not included in the table $S D$ standard deviation, TFC total functional capacity it was 'slightly important', 'somewhat important', or 'very important' to manage chorea (Fig. 2a).

Respondents who reported that managing chorea was at least 'Slightly Important' were also asked an open-ended question about why it was important to manage chorea. Based on a review of themes among those who provided open-text responses $(n=65)$, loss of independence $(18 \%$, $n=7)$, unpredictability/uncontrollability $(18 \%, n=7)$, fear of chorea getting worse $(15 \%, n=6)$, fear of falling $(15 \%, n=6)$, painful/harmful $(15 \%, n=6)$, and impact on family life $(13 \%, n=5)$ were the main reasons found in the HD cohort $(n=40)$. In the caregiver cohort $(n=25)$, loss of independence $(28 \%, n=7)$, impact on daily activities $(16 \%, n=4)$, trouble walking $(16 \%, n=4)$, stability issues $(12 \%, n=3)$, and frequent falls $(12 \%$, $n=3$ ) were the main reasons. Respondents with chorea were also asked in an open-ended question to list the three aspects that most bothered them about chorea. The most common responses in the HD cohort $(n=40)$ were anxiety $(53 \%, n=21)$, trouble walking $(28 \%, n=11)$, jerking/ shaking/kicking $(25 \%, n=10)$, trouble sleeping $(20 \%$, $n=8)$, falling ( $18 \%, n=7)$, memory/speech problems $(15 \%, n=6)$, movements in hands/feet $(15 \%, n=6)$, impact on daily activities $(13 \%, n=5)$, going out in public $(13 \%, n=5)$, and dropping or spilling $(13 \%, n=5)$. The most common responses in the caregiver cohort $(n=27)$ were trouble walking $(63 \%, n=17)$, anxiety $(30 \%$, $n=8)$, impact on social/family life $(22 \%, n=6)$, trouble eating/swallowing/choking $(22 \%, n=6)$, trouble getting dressed/bathing $(19 \%, n=5)$, and falling $(15 \%, n=4)$.
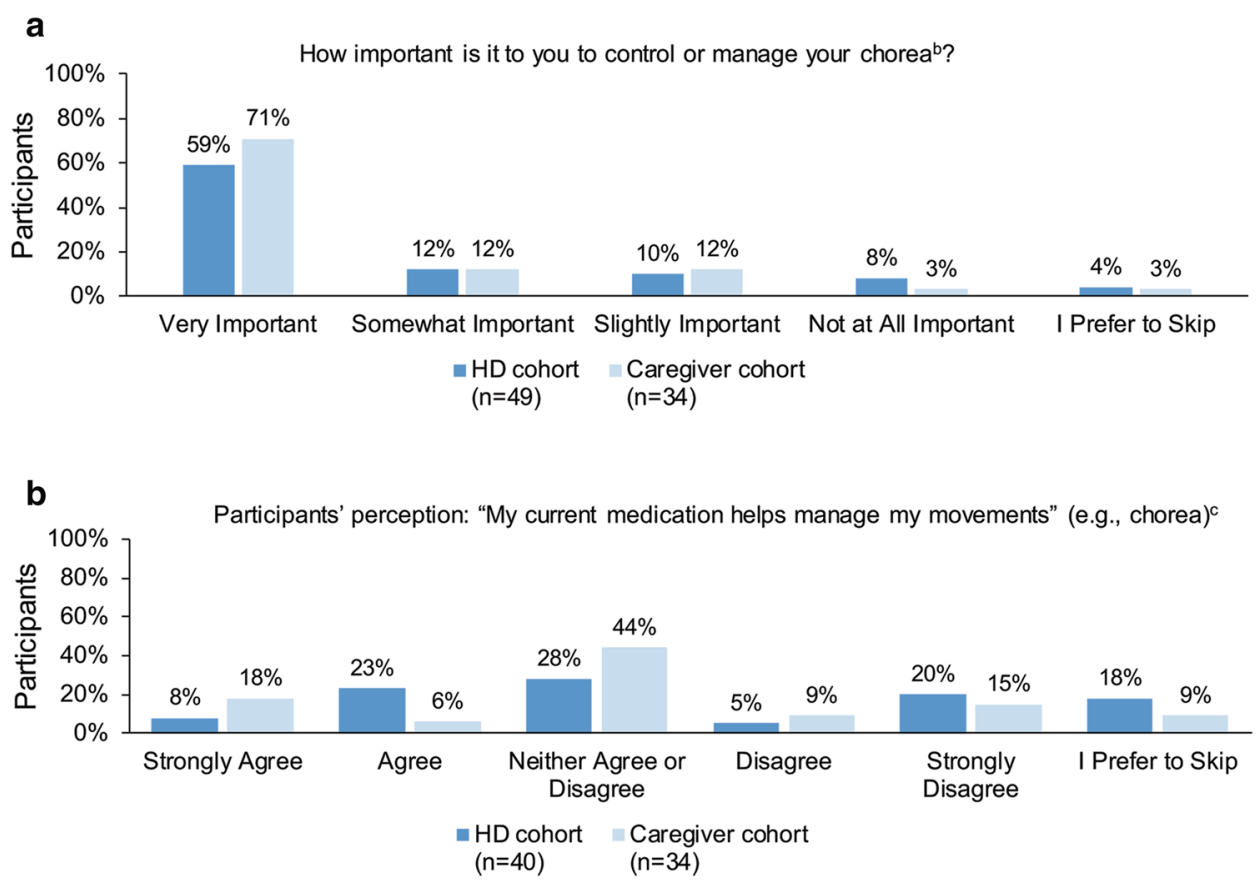

Fig. 2 Perception of treatment to control chorea ${ }^{a}$. a Participants responded to a 4-point Likert scale question 'How important is it to you to control or manage your chorea?' with an additional choice 'I Prefer to Skip'. b Participants' perception of a 5-point Likert scale question 'My current medication helps manage my movements' with an option to skip the question. These participants $(n=74)$ were among those who reported having experienced chorea $(n=83)$, and they may take medications not prescribed for HD. ${ }^{\mathbf{a}}$ Percentages may not sum to $100 \%$ due to rounding. ${ }^{b}$ Three individuals responded, 'I don't experience chorea', but had indicated in a previous question that they do experience chorea. These three respondents were excluded post hoc from follow-up questions pertaining to chorea. ${ }^{\mathrm{c}}$ Deutetrabenazine (Austedo ${ }^{\circledR}$ ) was not approved for the treatment of chorea at the time of the study. $H D$ Huntington disease 
Descriptions of themes identified and representative quotes are provided in Electronic Supplementary Material Table 2.

\subsubsection{Patient Experience with Chorea Treatment}

Less than half of respondents reported taking a medication to treat $\mathrm{HD}(45 \%, n=52)$; of those, less than half reported being either 'somewhat satisfied' $(19 \%, n=10)$, 'very satisfied' (23\%, $n=12)$, or 'extremely satisfied' (6\%, $n=3$ ) with the medications used to treat HD.

Among the respondents who experienced chorea, 31\% in the HD cohort and 24\% in the caregiver cohort 'Agreed' or 'Strongly Agreed' that the medication helped manage chorea (Fig. 2b). Patients were asked to select from a list of medications all those they were currently taking for HD. This list included: tetrabenazine $\left(\right.$ Xenazine $^{\circledR}$ ), clonazepam $\left(\right.$ Klonopin $^{\circledR}$, Genentech USA Inc., South San Francisco, CA, USA), risperidone (Risperdal ${ }^{\circledR}$, Janssen Pharmaceuticals Inc., Titusville, NJ, USA), sertraline $\left(\right.$ Zoloft $^{\circledR}$, Pfizer Inc., New York, NY, USA), escitalopram (Lexapro ${ }^{\circledR}$, Allergan USA, Inc., Irvine, CA, USA), bupropion (Wellbutrin $^{\circledR}$, GlaxoSmithKline, Research Triangle Park, NC, USA), citalopram (Celexa ${ }^{\circledR}$, Forest Pharmaceuticals Inc., St Louis, MO, USA), fluoxetine (Prozac ${ }^{\circledR}$, Eli Lilly and Company, Indianapolis, IN, USA), olanzapine (Zyprexa ${ }^{\circledR}$, Eli Lilly and Company), venlafaxine (Effexor ${ }^{\circledR}$, Pfizer), haloperidol (Haldol ${ }^{\circledR}$, Janssen-Cilag Ltd, High Wycombe, UK), fluphenazine (Prolixin ${ }^{\circledR}$, APOTHECON $^{\circledR}$, Princeton, NJ 08540 USA), paroxetine (Paxil ${ }^{\circledR}$, GlaxoSmithKline), and 'Other (please specify)'. The most frequently reported response was the "Other" category (38\% of $n=52$ currently taking a prescription medication for HD) followed by tetrabenazine (29\% of $n=52$ currently taking a prescription medication for HD). At the time of the survey, tetrabenazine was the only FDA-approved medication for HD chorea. Of the 25 people with HD who have ever taken tetrabenazine (combination of patients with current or previous exposure to tetrabenazine), ten $(40 \%)$ were satisfied ('somewhat satisfied', 'very satisfied', or 'extremely satisfied') with the medication; however, these numbers were too low for further analysis. When asked about adverse effects from this medication, fatigue was the most frequently reported $(n=12)$, followed by depression, feelings of restlessness, sleepiness $(n=8$ each), anxiety $(n=7)$, and irritability $(n=7)$.

\subsubsection{Patient DPMD Score}

Survey respondents were also asked a series of questions about their level of interest in participating in conversations with their providers around treatment and their overall health. The distribution of results among patient respondents from the DPMD are provided in Fig. 3. Across all items, patients in the HD cohort most frequently indicated that it was 'very important' or 'more important than anything else' for them to participate in discussions around their treatment and care, including doctors listening to them (95\%), knowing all the possible ways to treat HD (95\%), and receiving explanations of physician's recommendations (94\%) (Fig. 3a). In the caregiver cohort, all participants indicated that it was 'very important' or 'more important than anything else' to know all the possible ways to treat the condition $(100 \%)$, for the doctor to provide all the information needed to care for HD (100\%), and to tell the doctor what was concerning about the treatment plan (100\%) (Fig. 3b).

\subsection{Impact of Chorea on Health-Related Quality of Life and DPMD}

\subsubsection{HDQLIFE Chorea}

Among the entire study population, the average chorea domain score of the HDQLIFE was 59.3 (Fig. 4a). This observation varied by disease stage; participants with laterstage HD (stages IV and V, according to TFC guidelines) had, on average, higher Chorea domain scores (61.3) than those with early-stage HD (54.2) (Fig. 4b).

\subsubsection{Anxiety and Chorea}

As previously noted, several measures from Neuro-QoL and PROMIS were included in the study. Neuropsychiatric symptoms, as measured by the Anxiety score, as well as other symptoms, measured by the Stigma and Positive Affect scores, also differed significantly based on the extent to which chorea impacted quality of life (HDQLIFE Chorea). Significantly higher Anxiety scores were observed among those with high chorea than among those with low chorea \{mean [standard error (SE)]: 64.9 [1.1] vs. 60.8 [1.7], respectively; $P=0.0423$ \} (Fig. 5a). Further review indicated that anxiety was slightly, but significantly, correlated with the Chorea score (correlation coefficient $r=0.25, P=0.0222$ ), suggesting that increasing levels of anxiety were associated with an increasing impact of chorea on HRQoL.

\subsubsection{Stigma and Chorea}

Similarly, significantly higher Stigma scores were observed among individuals with high chorea than in those with low chorea [mean (SE): 60.9 (1.0) and 50.5 (1.3), respectively; $P \leq 0.0001$; Fig. 5b]. A positive linear association was also observed between stigma and Chorea scores (correlation coefficient $r=0.66, P<0.0001$ ), suggesting that having a 
a

How Important is it to you... (HD cohort, $n=80$ )

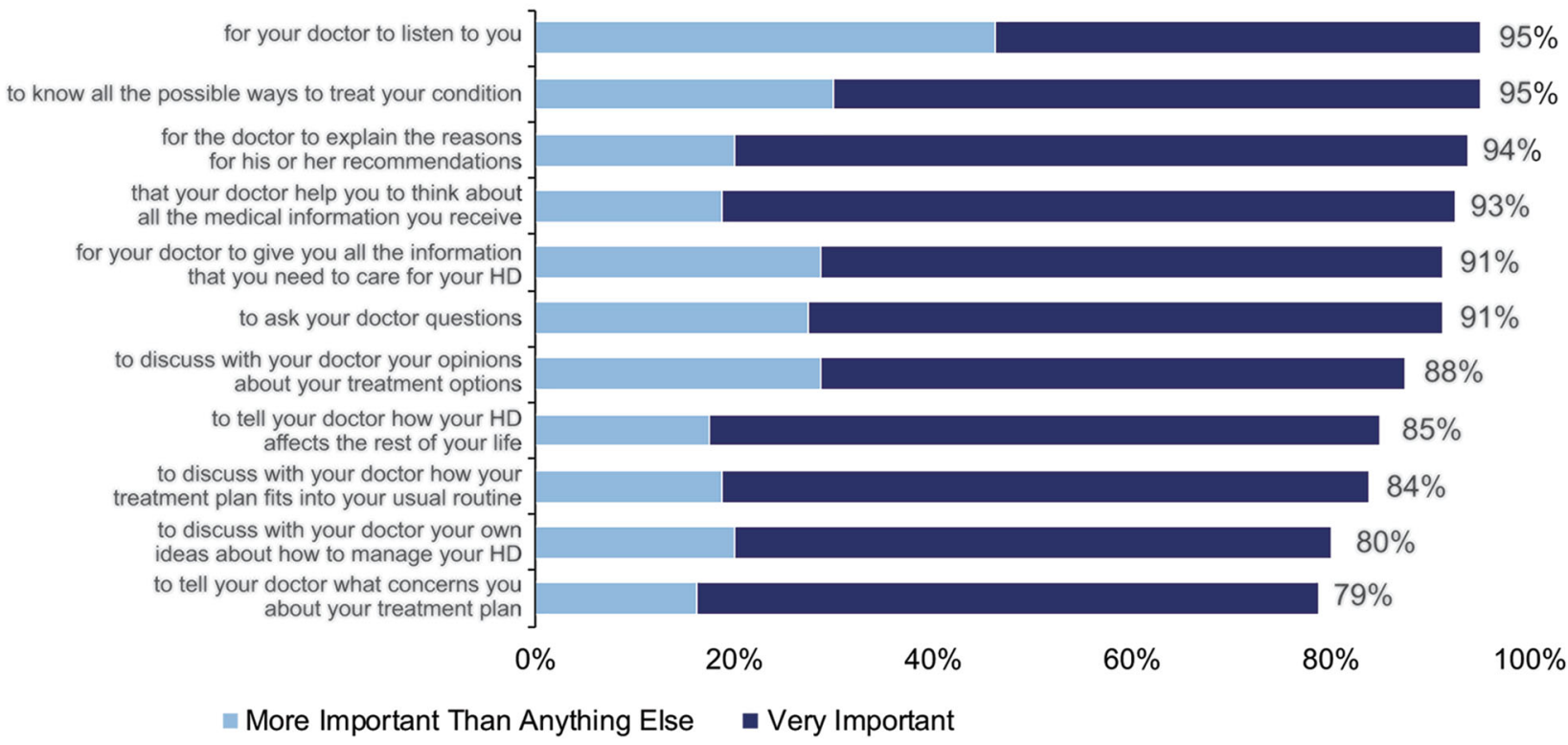

b

How Important is it to you... (caregiver cohort, $\mathrm{n}=35$ )

to know all the possible ways to treat your condition

for your doctor to give you all the information that you need to care for your HD to tell your doctor what concerns you about your treatment plan for the doctor to explain the reasons for his or her recommendations that your doctor help you to think about all the medical information you receive to ask your doctor questions for your doctor to listen to you to discuss with your doctor your opinions about your treatment options to tell your doctor how your $\mathrm{HD}$ affects the rest of your life to discuss with your doctor how your treatment plan fits into your usual routine

to discuss with your doctor your own ideas about how to manage your HD

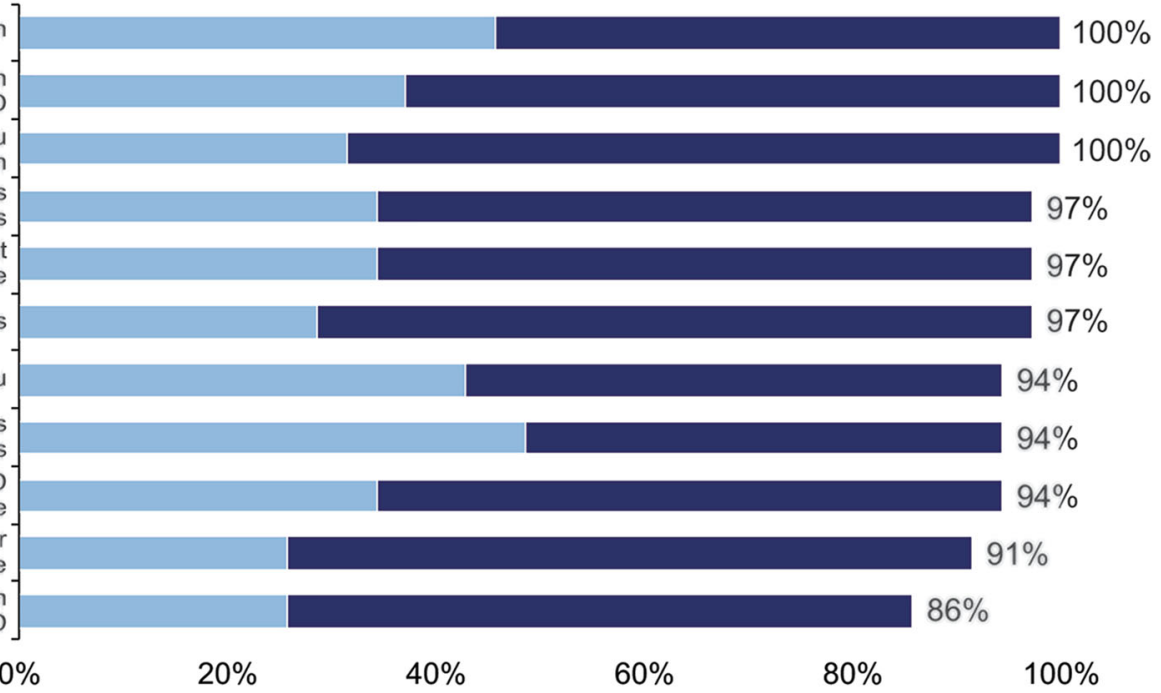

- More Important Than Anything Else $\quad$ Very Important

Fig. 3 DPMD among patient survey respondents ${ }^{\mathrm{a}}$. Participants in the HD cohort responded to 4-point Likert scale questions that assessed their desire to participate in medical decision-making. Percentages of participants who responded 'very important' (blue bars) or 'more

greater perception of social stigma was associated with an increasing impact of chorea on HRQoL. important than anything else' (gray bars) are shown as data labels. $D P M D$ desire to participate in medical decision-making, $H D$ Huntington disease

\subsubsection{Positive Affect and Chorea}

The reverse pattern was observed when reviewing Positive Affect stratified by Chorea scores. Significantly higher Positive Affect scores were observed among participants 
a HDQLIFE Chorea Score Distribution $(n=83)$

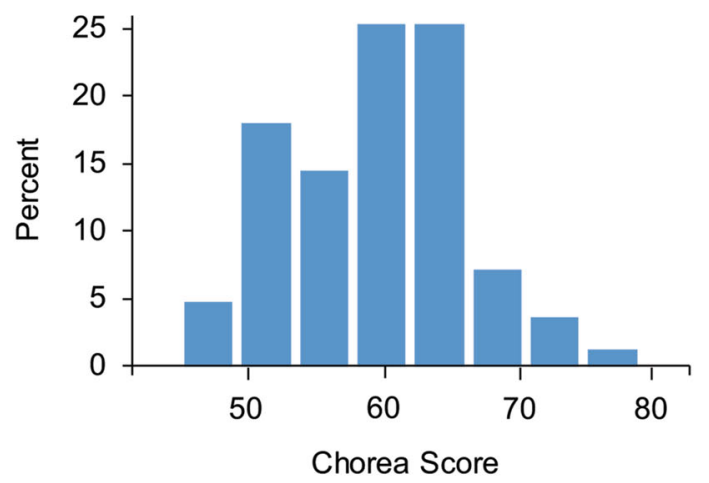

b

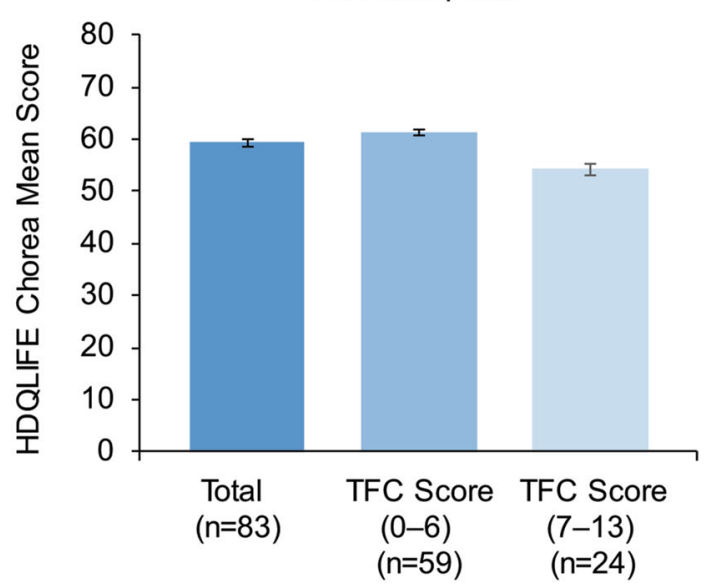

Fig. 4 HDQLIFE chorea score distribution and score by TFC. a HDQLIFE Chorea score distribution (\%). b HDQLIFE Chorea scores by TFC score in all participants. Error bars represent standard error of the mean. $H D$ Huntington disease, TFC total functional capacity

with low chorea than those with high chorea [mean (SE): 50.1 (1.2) and 44.0 (1.1), respectively; $P=0.0004$ ] (Fig. 5c). Moreover, a negative linear association was observed between Positive Affect and Chorea scores (correlation coefficient $r=-0.48, P<0.0001$ ), suggesting a less positive outlook among individuals who reported an increasing impact of chorea on physical and social functioning.

\subsubsection{DPMD and Chorea}

Individuals with HD and high chorea also had a greater desire to participate in medical decision-making, as assessed by DPMD score [28], than those with low chorea [mean (SE): 68.6 (2.5) vs. 60.4 (3.6)], although the difference was non-significant $(P=0.0612)$ (Fig. 5d).

\subsubsection{Regression Analysis}

Further evaluation of the effect of self-reported TFC and other HDQLIFE measures on HDQLIFE Chorea based on a multivariate linear regression model indicated agreement with associations observed based on descriptive stratified subgroup analyses and correlation results. The model explained $61 \%$ of the variability observed for the Chorea score (adjusted $R^{2}=0.61$ ) among survey respondents. As scores for Stigma increased (i.e., worsened Stigma), Chorea scores also increased $(P=0.0031)$. Self-reported TFC was also significantly associated with the impact of chorea on HRQoL: for every 1 unit increase in self-reported TFC scores (i.e., less severe disease stage), Chorea scores decreased by a factor of 0.90 . As positive affect scores increased (1 unit increase), chorea scores decreased by a factor of $-0.16(P=0.0149)$. The association between chorea and anxiety was no longer significant when controlling for other variables in the model, possibly due to many other factors that might be related to anxiety and because the relationship between anxiety and the impact of chorea on HRQoL is potentially bi-directional (Table 3).

\section{Discussion}

In the current study, most individuals with HD were experiencing chorea at the time of survey and most participants indicated that it was 'very important' to manage this symptom. Those experiencing chorea had psychological burdens such as stigma, loss of independence, fear of their symptoms worsening, fear of falling, and anxiety. They also reported functional impairment such as difficulty walking, difficulty sleeping, and challenges with mobility, such as driving. Individuals with more advanced disease had more severe problems, with higher levels of anxiety and perceived stigma causing decreased HRQoL. Conversely, those with higher ratings of 'Positive Affect' had better HRQoL. Chorea may lead to embarrassment and shame (e.g., social stigma), and both the physical and psychological aspects may make patients experience low confidence and restrict them from participating in social or leisure activities.

Despite the clear unmet need, less than half of the participants in our study were taking any medication to treat chorea. Among those taking such medication, only a quarter of patients were 'very satisfied' or 'extremely satisfied' with their medication. Less than half of those treated 'agreed' or 'strongly agreed' that the medication helped manage HD. Less than a quarter of patients who experienced chorea were taking tetrabenazine, the only FDAapproved medication to treat HD chorea at the time of the survey. Although participants were not asked why they 

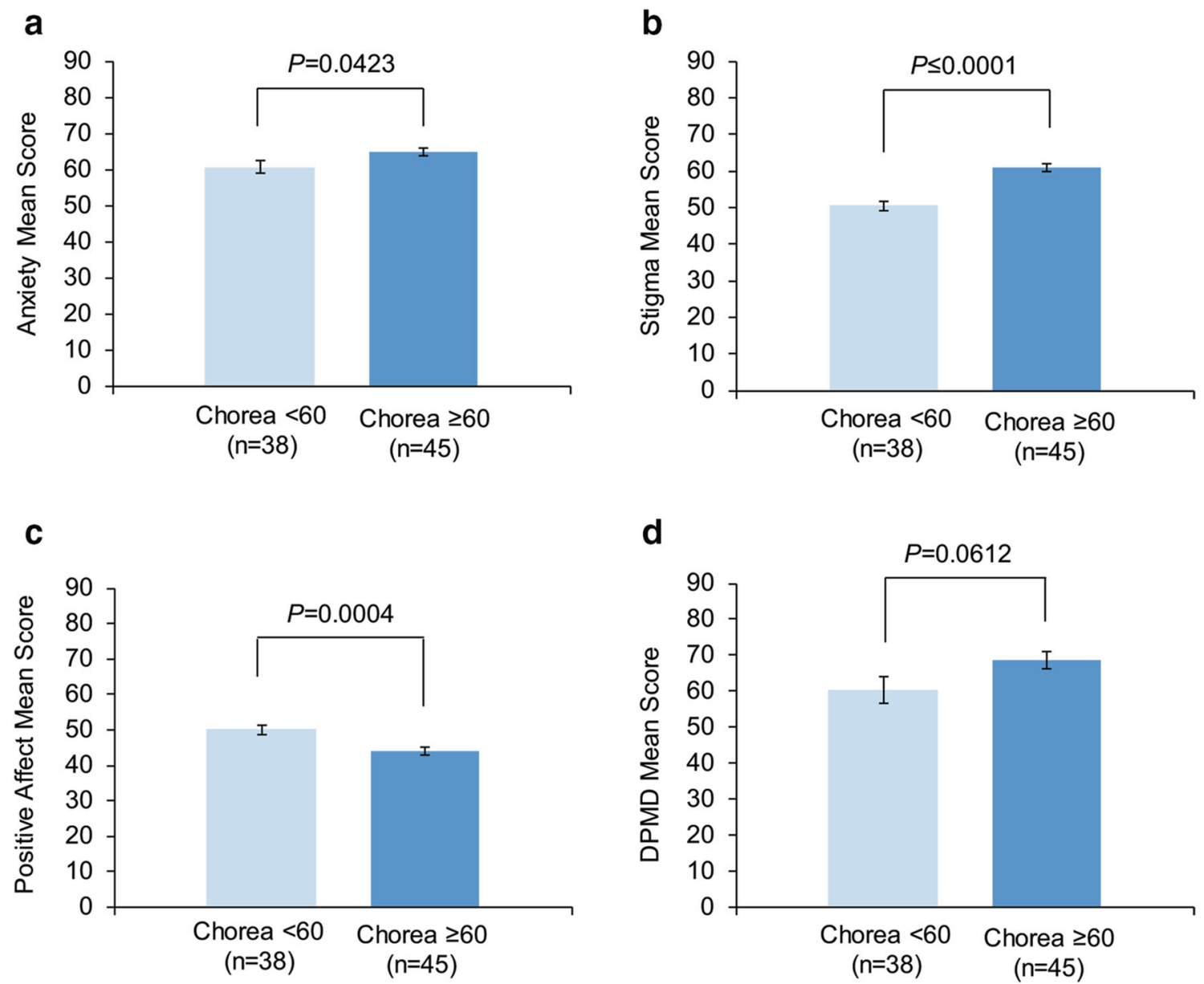

Fig. 5 a Anxiety, b stigma, c positive affect, d DPMD scores by Chorea subgroups. Error bars represent standard error of the mean. DPMD desire to participate in medical decision-making

Table 3 Relationship between HDQLIFE Chorea score and Patient-Reported Outcomes Measurement Information System (PROMIS)/Quality of Life in Neurological Disorders (Neuro-QoL) measures or total functional capacity

\begin{tabular}{|c|c|c|c|c|}
\hline Covariate $(n=83)$ & Regression coefficient $\beta$ & $95 \% \mathrm{CI}$ & SE & $P$ value \\
\hline TFC & -0.903 & -1.217 to -0.590 & 0.158 & $<0.0001$ \\
\hline Stigma & 0.217 & 0.075 to 0.359 & 0.0713 & 0.0031 \\
\hline Anxiety & -0.052 & -0.167 to 0.062 & 0.0574 & 0.3657 \\
\hline Positive affect & -0.1641 & -0.295 to 0.033 & 0.066 & 0.0149 \\
\hline
\end{tabular}

$C I$ confidence interval, $S E$ standard error, $T F C$ total functional capacity

were not taking medication to manage their chorea, possible reasons might include clinicians' concern about worsening depression or suicidality and no strong evidence supporting robust antichoreic effects of neuroleptic medications [14]. These results are consistent with another study, including 134 patients attending a regional HD neurogenetic clinic, which showed that $27 \%$ of HD patients were not taking any medication to manage behavioral or motor symptoms [36]. Limited evidence is available to support dosing and finding the safest and most effective combinations of drugs to manage HD because psychiatric and behavioral symptoms can be quite complex [14, 37]. This lack of effective and safe treatment for HD may contribute to compromised HRQoL. The results of this survey indicate a desire among patients to participate in medical decision-making, especially among those with the lowest HRQoL. While these findings may be complicated by the nature of the disease progression, patient and 
caregiver feedback in combination with assessments such as HDQLIFE Chorea can potentially help monitor disease progression and promote communication among patients, caregivers, and physicians. This study also demonstrates the need to consider contextual emotional and psychosocial factors during the assessment of chorea. A recent survey of physical therapists reported under-referral to supportive services that can help prevent falls, provide useful equipment, and promote lifestyle adjustments for the management of chorea and other motor symptoms [38]. Harnessing these resources could help patients and support caregivers with minimal risk.

The survey used in this study has a number of strengths and limitations. Strengths included the fact that, as an online study, we were able to gather data from 115 people with HD in only 6 weeks. Respondents could participate anonymously from their home at a time of their choice, which may have minimized potential social desirability bias. We used validated questionnaires (e.g., HDQLIFE) [29] with known psychometric qualities. However, in terms of limitations, an online survey requires that patients have sufficient cognitive ability to use a computer, which might limit responses from more impaired patients. Although the sample was generated relatively quickly (6 weeks), there are larger studies in the literature, such as the HDQLIFE development study, which recruited 536 participants, versus 115 respondents from this study. All data were crosssectional and self-reported, without independent confirmation from a physician or medical records. Therefore, the reported perceptions of patients may deviate from their actual experiences due to unawareness or diminished awareness of motor, cognitive, behavioral, and functional aspects of HD [39]. In addition, our focus on chorea may have excluded other important issues for patients, such as cognitive and psychiatric symptoms. Most of the participants in this study were white/Caucasian $(96 \%)$ or female (67\%). Such biases toward higher percentages of white or female participants are typical of respondents to online surveys (e.g., PatientsLikeMe) and other studies [40-42]. Therefore, the results may not be generalizable to minority populations. Relative to other studies in the literature suggesting that approximately $90 \%$ of HD patients experience chorea [7, 9], our sample showed a lower percentage (74\%). Although it cannot be ruled out that this is an unrepresentative sample, this sample might reflect a healthier population of patients. Alternatively, some respondents may have had confusion in distinguishing chorea from other symptoms such as tics or ataxia, which can be a problem for both patients and clinicians [6]. Future studies could address these issues by incorporating objective measurements from smartphones or wearable devices.

\section{Conclusions}

Individuals with HD and their caregivers consider the management of HD-related chorea to be important, as it negatively impacts HRQoL and overall functioning. However, better chorea treatment options are needed to successfully manage symptoms and help improve HRQoL. When evaluating the impact of chorea on HRQoL among patients, anxiety, stigma, and their outlook on their wellbeing are important contextual factors that need to be considered in treatment plans.

Author Contributions EMT, NEC, and PW contributed to study concept and design, data collection, data analysis, interpretation of results, and drafting of the manuscript. EMT also acts as overall guarantor of this work. RGI, SKG, VA, and KEA contributed to study concept and design, and interpretation of results. CC contributed to data analysis and interpretation of results. All authors contributed to the review, critical revision, and final approval of the manuscript.

\section{Compliance with Ethical Standards}

Funding This study was funded by Teva Pharmaceutical Industries, Petach Tikva, Israel. We thank the patients and caregivers who participated in this study, and Yue Liu, PhD (Chameleon Communications International with funding from Teva Pharmaceutical Industries) for editorial assistance in the preparation of this manuscript.

Conflict of interest EMT, PW, and CC are employees of PatientsLikeMe. The PatientsLikeMe Research Team has received research funding (including conference support and consulting fees) from AbbVie, Accorda, Actelion, Alexion, Amgen, AstraZeneca, Avanir, Biogen, Boehringer Ingelheim, Celgene, EMD, Genentech, Genzyme, Janssen, Johnson \& Johnson, Merck, Neuraltus, Novartis, Otsuka, Permobil, Pfizer, Sanofi, Shire, Takeda, Teva, and UCB. The PatientsLikeMe Research and Development Team has received research grant funding from Kaiser Permanente, the Robert Wood Johnson Foundation, Sage Bionetworks, The AKU Society, and the University of Maryland. PW also holds stock options in the company. $\mathrm{He}$ is an associate editor at the Journal of Medical Internet Research and is on the Editorial Boards of the BMJ, BMC Medicine, and Digital Biomarkers. He has received speaker fees from Bayer and honoraria from Roche, ARISLA, AMIA, IMI, PSI, and the BMJ. RGI and SKG are employees of Teva Pharmaceutical Industries. VA was an employee of Teva Pharmaceuticals Industries at the time of the study. KEA: Scientific Advisor: North American study Co-Principal Investigator for LEGATO-HD, Global Principal Investigator for AIM-TD, and Global Co-Principal Investigator for ARM-TD. Site Principal Investigator for Pride-HD, First-HD, ARC-HD: Teva. Scientific Advisor, Site Principal Investigator for ENROLL-HD: CHDI Foundation. Scientific Advisor: Prana. Site Principal Investigator: Vaccinex. Consultant to the NeuroNext 105 study: Azevan, salary support from the Griffin Foundation. She has also received honoraria from Vindico Medical Education. NEC is an Associate Professor at the University of Michigan. She currently has research grants from the NIH, the Craig H. Neilsen Foundation and Teva Pharmaceuticals; she is also supported as a co-investigator by grant funding from the $\mathrm{NIH}$ and CHDI. She provides patient reported outcome measurement selection and application consultation for Teva Pharmaceuticals. 
Ethics approval The study was reviewed by the New England Institutional Review Board (NEIRB) and designated a minimal-risk study; therefore, it was exempted from further review. Participants were provided with an informed consent form (approved by the NEIRB), which had to be understood and agreed upon prior to any data collection. All procedures performed in studies involving human participants were in accordance with the ethical standards of the institutional and/or national research committee and with the 1964 Helsinki Declaration and its later amendments or comparable ethical standards.

Informed consent Informed consent was obtained from all individual participants included in the study.

Data availability The datasets generated and/or analyzed during the current study are available from the corresponding author on reasonable request.

Open Access This article is distributed under the terms of the Creative Commons Attribution-NonCommercial 4.0 International License (http://creativecommons.org/licenses/by-nc/4.0/), which permits any noncommercial use, distribution, and reproduction in any medium, provided you give appropriate credit to the original author(s) and the source, provide a link to the Creative Commons license, and indicate if changes were made.

\section{References}

1. Foroud T, Gray J, Ivashina J, Conneally PM. Differences in duration of Huntington's disease based on age at onset. J Neurol Neurosurg Psychiatry. 1999;66:52-6.

2. Jankovic J. Treatment of hyperkinetic movement disorders. Lancet Neurol. 2009;8:844-56. https://doi.org/10.1016/S14744422(09)70183-8.

3. Ross CA, Aylward EH, Wild EJ, Langbehn DR, Long JD, Warner $\mathrm{JH}$, et al. Huntington disease: natural history, biomarkers and prospects for therapeutics. Nat Rev Neurol. 2014;10:204-16. https://doi.org/10.1038/nrneurol.2014.24.

4. Ho AK, Hocaoglu MB, European Huntington's Disease Network Quality of Life Working Group. Impact of Huntington's across the entire disease spectrum: the phases and stages of disease from the patient perspective. Clin Genet. 2011;80:235-9. https://doi. org/10.1111/j.1399-0004.2011.01748.x.

5. Carlozzi NE, Tulsky DS. Identification of health-related quality of life (HRQOL) issues relevant to individuals with Huntington disease. J Health Psychol. 2013;18:212-25. https://doi.org/10. 1177/1359105312438109.

6. Roos RAC. Huntington's disease: a clinical review. Orphanet Rare Dis. 2010;5:1-8. https://doi.org/10.1186/1750-1172-5-40.

7. Burgunder JM, Guttman M, Perlman S, Goodman N, van Kammen DP, Goodman L. An international survey-based algorithm for the pharmacologic treatment of chorea in Huntington's disease. PLoS Curr. 2011;3:RRN1260. https://doi.org/10.1371/ currents.RRN1260.

8. Fritz NE, Rao AK, Kegelmeyer D, Kloos A, Busse M, Hartel L, et al. Physical therapy and exercise interventions in Huntington's disease: a mixed methods systematic review. J Huntingtons Dis. 2017;6:217-35. https://doi.org/10.3233/JHD-170260.

9. Anderson KE. Huntington's disease. Handb Clin Neurol. 2011;100:15-24. https://doi.org/10.1016/B978-0-444-52014-2. 00002-1.

10. XENAZINE ${ }^{\mathrm{TM}}$ (tetrabenazine) (prescribing information). Deerfield, IL: Lundbeck; 2017. Available at: http://www.lundbeck.
com/upload/us/files/pdf/Products/Xenazine_PI_US_EN.pdf. Accessed April 2018.

11. Huntington Study Group. Tetrabenazine as antichorea therapy in Huntington disease: a randomized controlled trial. Neurology. 2006;66:366-72. https://doi.org/10.1212/01.wnl.0000198586. 85250.13

12. AUSTEDO ${ }^{\mathrm{TM}}$ (deutetrabenazine) tablets [prescribing information]. North Wales, PA: Teva Pharmaceuticals USA, Inc. 2017. Available at: https://www.accessdata.fda.gov/drugsatfda_docs/ label/2017/208082s000lbl.pdf. Accessed April 2018.

13. Teva Pharmaceutical Industries [press release]. Teva announces FDA approval of AUSTEDO ${ }^{\mathrm{TM}}$ (deutetrabenazine) tablets for the treatment of chorea associated with Huntington's disease. 2017. http://www.tevapharm.com/news/teva_announces_fda_approval_ of_austedo_deutetrabenazine_tablets_for_the_treatment_of_ chorea_associated_with_huntington_s_disease_04_17.aspx. Accessed 2 May 2018.

14. Armstrong MJ, Miyasaki JM. Evidence-based guideline: pharmacologic treatment of chorea in Huntington disease: report of the guideline development subcommittee of the American Academy of Neurology. Neurology. 2012;79:597-603. https:// doi.org/10.1212/WNL.0b013e318263c443.

15. Chapuis S, Ouchchane L, Metz O, Gerbaud L, Durif F. Impact of the motor complications of Parkinson's disease on the quality of life. Mov Disord. 2005;20:224-30. https://doi.org/10.1002/mds. 20279.

16. Bandstra NF, Camfield CS, Camfield PR. Stigma of epilepsy. Can J Neurol Sci. 2008;35:436-40.

17. Grytten N, Maseide P. 'When I am together with them I feel more ill'. The stigma of multiple sclerosis experienced in social relationships. Chronic Illn. 2006;2:195-208. https://doi.org/10.1177/ 17423953060020030101.

18. Aubeeluck AV, Buchanan H, Stupple EJ. 'All the burden on all the carers': exploring quality of life with family caregivers of Huntington's disease patients. Qual Life Res. 2012;21:1425-35. https://doi.org/10.1007/s11136-011-0062-x.

19. Banaszkiewicz K, Sitek EJ, Rudzinska M, Soltan W, Slawek J, Szczudlik A. Huntington's disease from the patient, caregiver and physician's perspectives: three sides of the same coin? J Neural Transm (Vienna). 2012;119:1361-5. https://doi.org/10.1007/ s00702-012-0787-x.

20. Wicks P, Massagli M, Frost J, Brownstein C, Okun S, Vaughan $\mathrm{T}$, et al. Sharing health data for better outcomes on PatientsLikeMe. J Med Internet Res. 2010;12:e19. https://doi.org/10. 2196/jmir.1549.

21. Hixson JD, Barnes D, Parko K, Durgin T, Van Bebber S, Graham A, et al. Patients optimizing epilepsy management via an online community: the POEM study. Neurology. 2015;85:129-36. https://doi.org/10.1212/WNL.0000000000001728.

22. DA Belsley KE, Welsch RE. Regression diagnostics: identifying influential data and sources of collinearity. New York: John Wiley; 1980.

23. Hsieh HF, Shannon SE. Three approaches to qualitative content analysis. Qual Health Res. 2005;15:1277-88. https://doi.org/10. $1177 / 1049732305276687$.

24. Carlozzi NE, Boileau N, Perlmutter JS, et al. Agreement between clinician-rated versus patient-reported outcomes in Huntington disease. J Neurol. 2018. https://doi.org/10.1007/s00415-0188852-5 (Epub 2018 Apr 23).

25. Shoulson I. Huntington disease: functional capacities in patients treated with neuroleptic and antidepressant drugs. Neurology. 1981;31:1333-5.

26. Dubinsky R. The Huntington's disease Workgroup of Promoting Excellence in End-of-Life Care Total Functional Capacity Scale. Available at: http://promotingexcellence.growthhouse.org/ huntingtons/monograph/pe5670.html. Accessed April 2018. 
27. Marder K, Zhao H, Myers RH, Cudkowicz M, Kayson E, Kieburtz K, et al. Rate of functional decline in Huntington's disease. Huntington Study Group. Neurology. 2000;54:452-8.

28. Golin CE, DiMatteo MR, Leake B, Duan N, Gelberg L. A diabetes-specific measure of patient desire to participate in medical decision making. Diabetes Educ. 2001;27:875-86. https://doi.org/ 10.1177/014572170102700613.

29. Carlozzi NE, Schilling SG, Lai JS, Paulsen JS, Hahn EA, Perlmutter JS, et al. HDQLIFE: development and assessment of health-related quality of life in Huntington disease (HD). Qual Life Res. 2016;25:2441-55. https://doi.org/10.1007/s11136-0161386-3.

30. Carlozzi NE, Downing NR, Schilling SG, Lai JS, Goodnight SM, Miner JA, et al. The development of a new computer adaptive test to evaluate chorea in Huntington disease: HDQLIFE Chorea. Qual Life Res. 2016;25:2429-39. https://doi.org/10.1007/s11136016-1307-5.

31. Carlozzi NE, Ready RE, Frank S, Cella D, Hahn EA, Goodnight SM, et al. Patient-reported outcomes in Huntington's disease: Quality of Life in Neurological Disorders (Neuro-QoL) and Huntington's disease Health-Related Quality of Life (HDQLIFE) physical function measures. Mov Disord. 2017;32:1096-102. https://doi.org/10.1002/mds.27046.

32. Cella D, Nowinski C, Peterman A, Victorson D, Miller D, Lai JS, et al. The neurology quality-of-life measurement initiative. Arch Phys Med Rehabil. 2011;92:S28-36. https://doi.org/10.1016/j. apmr.2011.01.025.

33. Gershon RC, Lai JS, Bode R, Choi S, Moy C, Bleck T, et al. Neuro-QOL: quality of life item banks for adults with neurological disorders: item development and calibrations based upon clinical and general population testing. Qual Life Res. 2012;21:475-86. https://doi.org/10.1007/s11136-011-9958-8.

34. Cella D, Riley W, Stone A, Rothrock N, Reeve B, Yount S, et al. The Patient-Reported Outcomes Measurement Information System (PROMIS) developed and tested its first wave of adult self- reported health outcome item banks: 2005-2008. J Clin Epidemiol. 2010;63:1179-94. https://doi.org/10.1016/j.jclinepi.2010. 04.011.

35. HealthMeasures Neuro-QoL. 2017. http://www.healthmeasures. net/explore-measurement-systems/neuro-qol. Accessed 28 Sep 2017.

36. Craufurd D, Thompson JC, Snowden JS. Behavioral changes in Huntington disease. Neuropsychiatry Neuropsychol Behav Neurol. 2001;14:219-26.

37. Reilmann R. Pharmacological treatment of chorea in Huntington's disease-good clinical practice versus evidence-based guideline. Mov Disord. 2013;28:1030-3. https://doi.org/10.1002/ mds. 25500 .

38. Busse ME, Khalil H, Quinn L, Rosser AE. Physical therapy intervention for people with Huntington disease. Phys Ther. 2008;88:820-31. https://doi.org/10.2522/ptj.20070346.

39. McCusker E, Loy CT. The many facets of unawareness in huntington disease. Tremor Other Hyperkinet Mov (N Y). 2014;4:257. https://doi.org/10.7916/D8FJ2FD3.

40. Tabrizi SJ, Langbehn DR, Leavitt BR, Roos RAC, Durr A, Craufurd D, et al. Biological and clinical manifestations of Huntington's disease in the longitudinal TRACK-HD study: cross-sectional analysis of baseline data. Lancet Neurol. 2009;8:791-801. https://doi.org/10.1016/S1474-4422(09)70170$\mathrm{X}$.

41. Pringsheim T, Wiltshire K, Day L, Dykeman J, Steeves T, Jette $\mathrm{N}$. The incidence and prevalence of Huntington's disease: a systematic review and meta-analysis. Mov Disord. 2012;27:1083-91. https://doi.org/10.1002/mds.25075.

42. Huntington Study Group, Frank S, Testa CM, Stamler D, Kayson E, Davis $\mathrm{C}$ et al. Effect of deutetrabenazine on chorea among patients with Huntington disease: a randomized clinical trial. JAMA. 2016;316:40-50. https://doi.org/10.1001/jama.2016. 8655. 